\title{
Evaluation of Oral and Dental Health Status in Hemophilic Children and Adolescents in the City of Rasht
}

\author{
(D) Katayoun Salem1, (1) Samad Seyyedkhamesi2, (1) Maedeh Aminian33
}

${ }^{1}$ Azad Dental School, Faculty of Dental, Department of Pediatric Dentistry, Tehran, Iran.

2Islamic Azad University Isfahan (khorasgan) Branch School of Dentistry, Department of Oral and Maxillofacial Surgery, Isfahan, Iran

${ }_{3}^{3}$ Isfahan University of Medical Science, Faculty of Dental, Department of Oral and Maxillofacial Radiology, Isfahan, Iran

\begin{abstract}
Aim: Patients with hemophilia need special care and attention in dentistry. Thus, we assessed the oral and dental health status in children and adolescents with hemophilia in Rasht, Iran.

Materials and Methods: In this case-control study, 53 hemophiliac patients (A and B), 2-15 years of age, and 53 healthy children were assessed. A checklist including demographic data was filled out and the subjects were examined by one dentist. The patients were assessed for modified gingival index, plaque index (PI) and Decayed-missing-filled teeth [(DMFT) in permanent teeth and Decayed-missing-filled teeth (dmft) in primary teeth)] indices. Data were analyzed with SPSS 19 at a significance level of $p<0.05$.

Results: The mean age of the subjects was $10.49 \pm 4.21$ years in the case group and $10.5 \pm 4.07$ years in the control group. $92.5 \%$ of the patients exhibited factor VIII deficiency and the most frequent blood group was A (34\%). $63.2 \%$ of the subjects in the hemophilia group and $13.2 \%$ in the control group had a history of bleeding that was significant $(p=0.0001)$. There were significant differences between the two groups in bleeding at tooth eruption and exfoliation sites of deciduous teeth $(p=0.0001)$, flossing $(p=0.046)$, toothpick use $(p=0.02)$ and the history of dental education ( $p=0.014)$. No significant difference was found between dmft and DMFT scores between the two groups but DMFS was significantly higher in the hemophilia group $(p=0.007)$. There was also a significantly higher Pl in the hemophilia group $(p=0.003)$.

Conclusion: Considering some unsatisfactory oral health conditions in these patients, dental health care and prevention seems necessary and can be effective in the prevention of dental problems, bleedings and complex treatments.

Keywords: Hemophilia, MGI index, DMFT index
\end{abstract}

\section{Introduction}

Treatment of patients with hereditary hemorrhagic conditions is associated with many problems. One of these conditions is hemophilia, which is the most common recessive $x$-linked hereditary hemorrhagic condition, with these affected patients comprising a sizeable proportion of patients with special conditions (1). This disease afflicts males at a ratio of 1:10.000 of the world population in all the ethnic groups (2). The prevalence of the disease is 1:9.800 of the population in Iran (3).

Hemorrhage from different areas of the oral cavity and gingival bleeding might be profuse and continue for a long time. Even the physiologic processes of tooth eruption and exfoliation might be associated with long periods of hemorrhage. Hemophilia pseudo-tumor in the mandible is a condition that might occur due to frequent sub-periosteal hemorrhage (4). Other oral manifestations, including a high 
rate of caries and widespread periodontal diseases, mostly appear to be secondary due to neglecting the oral cavity due to other systemic conditions and bleeding of oral tissues due to tooth-brushing. Oral ulcers are the common reason for hemorrhage in children with severe forms of hemophilia $(2,5)$. Intracranial hemorrhage, might leave serious sequelae and is one of the main etiologic factors for death in such children (6).

Patients with hemophilia require special attention and care during different dental procedures. Given the expenditure devoted to such patients annually, it is very important to follow different aspects of the health of such patients. Therefore, the present study was undertaken to evaluate the oral and dental health of children and adolescents with hemophilia in Rasht, Iran.

\section{Materials and Methods}

In the present descriptive-analytical study, all the 53 children and adolescents with hemophilia in Rasht, Iran, who were 2-15 years of age, were evaluated. In addition, a control group $(n=53)$ with no hemophilia, was selected from schoolchildren in Rasht, who were matched in relation to socio-economic status based on their parents' jobs and educational status, and age and gender with the hemophilia group.

A questionnaire was prepared by the dentist for both groups, which consisted of questions about their parents' educational level and job, history of bleeding in the oral cavity, the frequency of tooth-brushing, oral hygiene habits, history of routine dental visits, dental therapeutic needs and the history of regular fluoride therapy. The type of hemophilia, the factor level, the laboratory tests [human immunodeficiency virus (HIV), hepatitis C virus (HCV) antibody and hepatitis B virus surface (HBs) antigen] and the blood type of the hemophilia group were also extracted from the medical records of the patients.

Clinical evaluations for all the subjects were carried out by one operator while the patients were sitting on a chair based on the guidelines of the World Health Organization (7). Modified gingival index (MGI) and plaque index (PI) were used for the evaluation of periodontal status and Decayed-missing-filled teeth [(DMFT) in permanent teeth and Decayed-missing-filled teeth ( $\mathrm{dmft}$ ) in primary teeth] indices were used for the evaluation of dental caries in both groups.

DMFT and dmft indices were evaluated with the use of a blunt explorer and a flat dental mirror with the tactile and visual technique (4).

MGI (described by Loe and Silness) was used to evaluate gingival inflammation. To this end, examinations were carried out with the use of a flat mirror and observation of the gingiva with no dental probe. In this context, a five-score classification was used as follows:
Score 0 = no inflammation,

Score 1 = mild inflammation: minor changes in the color and consistency of the gingiva in all the gingival portions but not at all the gingival margins and interdental papillae,

Score 2 = mild inflammation: similar to score 1 but with the involvement of all the margins and papillae,

Score 3 = moderate inflammation: shining, rubor and edema or hypertrophy of the gingival margins or interdental papillae,

Score 4 = severe inflammation: clearly visible rubor, edema and hypertrophy of the marginal gingiva and interdental papillae in association with spontaneous bleeding, congestion and/or ulceration (4).

O'Leary PI was used to determine PI with the use of fuchsin plaque disclosing agent. After the patient has rinsed, the dentist examines the four surfaces of each tooth (except the occlusal surface) for accumulation of stain at the dentogingival junction. After all teeth are examined and scored, the index is calculated by dividing the number of plaque containing surfaces by the total number of available surfaces. (8).

\section{Statistical Analysis}

Data were analyzed with SPSS 19 at a significance level of $p<0.05$ with chi-squared test, Kolmogorov-Simonov test, Mann-Whitney $U$ test, Fisher's exact test and Kruskal-Wallis test.

\section{Ethical Approval}

All procedures performed in studies involving human participants were approved by the Ethics Committee of Dental School, Guilan University of Medical Sciences and with the 1964 Helsinki declaration and its later amendments or comparable ethical standards. Therefore, all the parents of the participants gave informed consent for inclusion in this study.

\section{Results}

The subjects consisted of 52 boys (98.1\%) and one girl $(1.9 \%)$ in each group. All the 53 patients with hemophilia were negative for HIV, HCV antibody and HBs antigen (which was not possible for the control group subjects).

Figure 1 presents the frequencies of bleeding in the oral cavity in both the control and hemophilia groups. Chi-squared test revealed that the history of bleeding in the whole oral cavity $(p=0.0001)$, tooth eruption site $(p=0.0001)$ and tooth exfoliation site $(p=0.0001)$ were significantly higher in the hemophilia groups.

Figure 2 presents the frequencies of oral and dental hygiene habits in the two groups. Chi-squared test revealed that the use of dental floss and toothpicks in the children and adolescents with hemophilia was significantly less frequent than that in the control group $(p=0.046, p=0.02)$. 


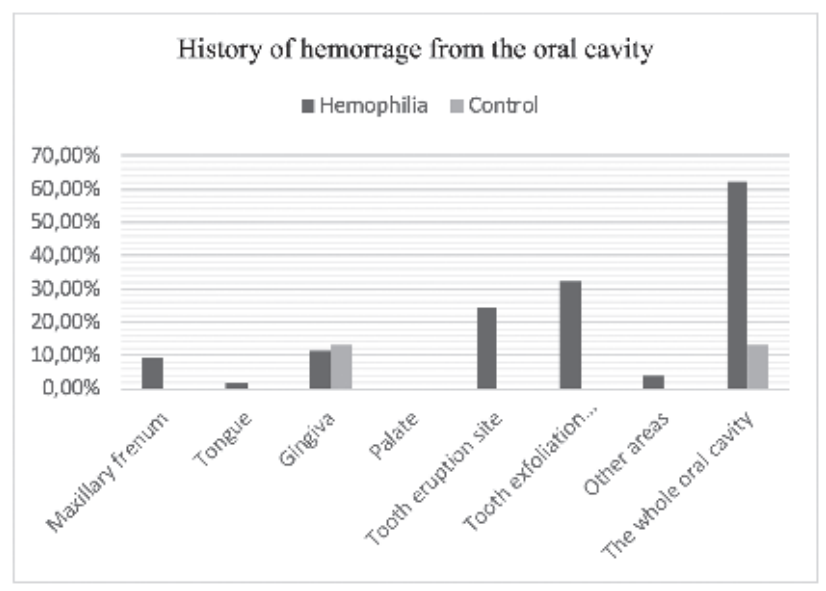

Figure 1. The frequencies of bleeding from the oral cavity in the control and hemophilia groups

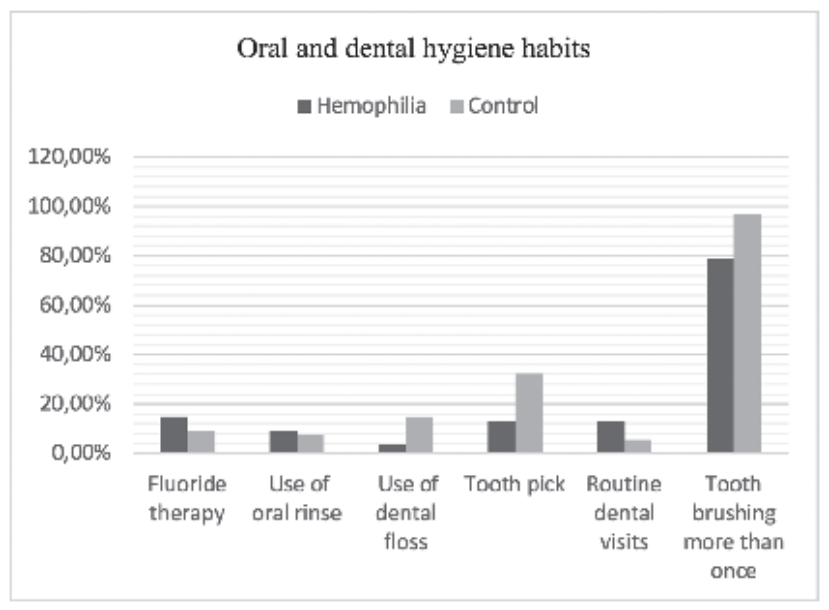

Figure 2. The frequencies of oral and dental hygiene habits in the two study groups

In addition, the frequency distributions of dental anomalies $(p=0.67)$, fluorosis $(p=0.49)$, hypoplasia $(p=1.0)$ and treatment needs $(p=0.49)$ were evaluated in the two groups and no significant differences were detected between them. $96.2 \%$ of the subjects in the control group and $100 \%$ of the patients with hemophilia required dental treatment. The most required services in the hemophilia group were caries-stopping care (83\%) and prevention (79.2\%) and in the control group were prevention (92.5\%) and caries-stopping care $(83 \%)$ respectively.

Table I compares the means of dmft, DMFT, Decayed Missing Filled Surface in permanent teeth (dmfs), Decayed Missing Filled Surface in permanent teeth (DMFS), PI and $\mathrm{MGl}$ between the two groups. Kolmogorov-Simonov test showed that none of the data of the parameters above were distributed normally in the two groups. Mann-Whitney $U$ test revealed that the means of DMFS and O'Leary index were significantly higher in the hemophilia group $(p=0.007$ and $\mathrm{p}=0.003)$
Comparison of the means of MGI, PI, DMFT and dmft separately for each hematologic factor in those children and adolescents with hemophilia is presented in Table II. Kolmogorov-Smirnov test showed that data in relation to DMFT and dmft and PI and MGI were not distributed normally in subjects with hemophilia $(p<0.05)$. In addition, Kruskal-Wallis test showed that only the difference in $\mathrm{MGI}$ was significant in terms of the hematologic factors $(p=0.011$.

Table III presents the frequency distributions of deficiencies of different factors and factor levels in children and adolescents with hemophilia in the present study. The greatest deficiency was related to factor VIII (92.5\%), followed by factor IX (5.7\%) and factor VII (1.8\%). Also, the most frequent factor level of deficiency was $<1 \%(52.8 \%)$, followed by $1-5 \%(26.4 \%)$ and $>5 \%(20.8 \%)$.

\section{Discussion}

The oral manifestations in patients with hemophilia appear in different forms. Hemorrhage from different parts of the oral cavity and from the gingiva might be profuse and continue for a long time. Even the physiologic processes of tooth eruption and exfoliation might be associated with long-term hemorrhage.

In the present study, the highest rate of hemorrhage in patients with hemophilia were related to the exfoliation of deciduous teeth and tooth eruption, followed by bleeding from the gingiva, maxillary frenum and tongue, in descending order.

Those subjects with hemophilia used brushes and dental floss at a significantly lower frequency compared to the control group, which might be due to fears of bleeding in the oral cavity; however, there were no significant differences between the two groups in relation to the use of mouthwashes, fluoride therapy and dental visits.

In addition, the dmfs index was not significantly different between the two groups, which might be attributed to the high prevalence rate of interproximal caries in deciduous teeth. However, the DMFS index in those children and

\begin{tabular}{|l|l|l|l|}
\hline \multicolumn{4}{|l|}{ Table I. The characteristics of the two study groups } \\
\hline & $\begin{array}{l}\text { Hemophilia } \\
\text { Mean } \pm \text { SD }\end{array}$ & $\begin{array}{l}\text { Control } \\
\text { Mean } \pm \text { SD }\end{array}$ & p value \\
\hline DMFT & $2.75 \pm 2.71$ & $1.98 \pm 2.51$ & 0.112 \\
\hline DMFS & $3.79 \pm 4.1$ & $2.03 \pm 4.05$ & 0.007 \\
\hline dmft & $2.83 \pm 3.06$ & $3.13 \pm 3.21$ & 0.66 \\
\hline dmfs & $4.9 \pm 6.2$ & $6.58 \pm 7.33$ & 0.25 \\
\hline The O'Leary index & $81.87 \pm 15.43$ & $72.78 \pm 18.94$ & 0.003 \\
\hline MGI & $1.86 \pm 0.8$ & $1.75 \pm 1.1$ & 0.3 \\
\hline
\end{tabular}

SD: Standard deviation, DMFT: Decayed-missing-filled teeth, MGI: Modified gingival index 
adolescents with hemophilia was significantly higher than that in the control group (3.79 vs. 2.3), indicating the presence of multi-surface caries in subjects with hemophilia, which might be attributed to the lower rate of use of dental floss and toothpicks.

A higher DMFS index in patients with hemophilia compared to the control group might be attributed to coagulation problems in patients with hemophilia; therefore, these patients prefer soft foods and liquids to decrease the risks of ulceration of the oral cavity mucosa. However, it should be considered that the mucosa in these subjects is not fragile and is only prone to bleeding; therefore, the best diet suggested for these patients is low-volume soft food with a high content of calorie and a low content of carbohydrates (2).

In a study by Salem and Eshghi (9), DMFT, DMFS, dmft and dmfs did not exhibit significant differences; however, there were more restored teeth in subjects with hemophilia compared to the control subjects. The differences between results of above mentioned study with ours, may probably attributed to lack of specialized dental care centers for Hemophilia patients in the city of Rash. As a result, they seek care into major Hemophilia dental clinics in other centers. Such centers and advanced facilities with free services for patients with hemorrhagic problems are available in Tehran to solve the health problems that these patients have in relation to their oral cavities.

In the present study, $92.5 \%$ of the patients with hemophilia had factor VIII deficiency and the rest had deficiencies of factors VII and IX. The factor levels were $<1 \%$ in $32.8 \%$ of the subjects (severe) and $1-5 \%$ in $26.7 \%$ of the subjects (moderate). However, in a study by Noor et al. (10), 75 subjects $(87 \%)$ had hemophilia A (47\% with mild form and $30 \%$ with severe form) and the rest had hemophilia B (55\% with mild form and $36 \%$ with severe form), indicating a higher prevalence of the severe form in Rasht in this age group.

In addition, in the present study, $96.2 \%$ of the subjects in the control group and $100 \%$ of the subjects with hemophilia required dental treatment, indicating poor oral hygiene in all groups in the society. Lack of attention to oral hygiene during childhood might result in dental and oral complications and morphological changes during adulthood, indicating the importance of paying more attention to dental care in all children. The best healthcare services in all the age groups, in both healthy children and children with special diseases, including hemophilia, should include attention to the prevention of dental diseases. Therefore, in addition to a decrease in the complications of oro-dental diseases in all age groups, the economic costs, time and morbidity in relation to diseases decrease significantly.

The PI in the patients in the hemophilia group might be explained by the way such patients brush their teeth and their avoidance of toothbrush contact with the gingiva and possible hemorrhage after brushing.

These patients comprise a small proportion of the population; however, they exert high treatment costs on the health systems (9).

Comprehensive hemophilic care consists of all the

Table III. Frequency distributions of deficiencies of different factors and factor levels in children and adolescents with hemophilia

\begin{tabular}{|l|l|l|l|l|l|}
\hline Factor & No. & Percentage & Factor level & No. & Percentage \\
\hline Factor VIII & 49 & 92.5 & $<1 \%$ & 28 & 52.8 \\
\hline Factor IX & 3 & 5.7 & $1-5 \%$ & 14 & 26.4 \\
\hline Factor VII & 1 & 1.8 & $>5 \%$ & 11 & 20.8 \\
\hline Total & 53 & 100 & Total & 53 & 100 \\
\hline
\end{tabular}

Table II. Comparison of the means of Decayed-missing-filled teeth, dmft, plaque index and Modified gingival index separately for the level of each hematologic factor in the children and adolescents with hemophilia

\begin{tabular}{|c|c|c|c|c|c|}
\hline & Factor level & No. & Mean & SD & $p$ value \\
\hline \multirow[t]{3}{*}{ DMFT } & $<1 \%$ & 28 & 2.35 & 2.45 & \multirow[t]{3}{*}{$p=0.173$} \\
\hline & $1-5 \%$ & 14 & 2.28 & 2.46 & \\
\hline & $>5 \%$ & 11 & 4.36 & 2.26 & \\
\hline \multirow[t]{3}{*}{$\mathrm{dmft}$} & $<1 \%$ & 28 & 2.75 & 2.8 & \multirow[t]{3}{*}{$p=0.122$} \\
\hline & $1-5 \%$ & 14 & 4.21 & 3.8 & \\
\hline & $>5 \%$ & 11 & 1.27 & 1.79 & \\
\hline \multirow[t]{3}{*}{ O'Leary index } & $<1 \%$ & 28 & 80.43 & 19.6 & \multirow[t]{3}{*}{$p=0.818$} \\
\hline & $1-5 \%$ & 14 & 83.61 & 7.43 & \\
\hline & $>5 \%$ & 11 & 83.32 & 10.4 & \\
\hline \multirow[t]{3}{*}{$\mathrm{MGI}$} & $<1 \%$ & 28 & 1.96 & 0.83 & \multirow[t]{3}{*}{$p=0.011$} \\
\hline & $1-5 \%$ & 14 & 2.14 & 0.66 & \\
\hline & $>5 \%$ & 11 & 1.27 & 0.64 & \\
\hline
\end{tabular}

SD: Standard deviation, DMFT: Decayed-missing-filled teeth, MGI: Modified gingival index 
medical services, including dental services. Prevention of dental problems is the main principle in oral care and might result in the avoidance of emergency events. Some of these procedures consist of brushing of the teeth with a fluoridated toothpaste, use of a soft or medium toothbrush, use of fluoride supplements, limiting the use of carbohydrates, use of non-cariogenic sugars and dental visits at least once a month for check-up, fluoride therapy and sealant therapy if necessary. At the same time, the health of periodontal tissues to prevent hemorrhage and tooth loss is of the utmost importance.

\section{Recommendations}

It is suggested that training sessions be held by the authorities for dentists in order to support the health of patients with special conditions, especially hemophilia. It is helpful to establish a special center for the oral health problems of hemophiliacs in Rasht in order to solve their problems in this respect. Also, ignoring these patients and lack of treatment for such patients by dentists all over Gilan Province due to problems in relation to long and un-controllable bleeding and avoiding such responsibility, due to fears about HIV and HBV etc. necessitate the establishment of a center to support these patients in Gilan Province.

\section{Ethics}

Ethics Committee Approval: The study was approved by Ethics Committee of Dental School, Guilan University of Medical Sciences (approval number: IR.GUMS.REC.1394.407).

Informed Consent: Consent form was filled out by all participants.

Peer-review: Externally and internally peer-reviewed.

\section{Authorship Contributions}

Surgical and Medical Practices: K.S., S.S., Concept: K.S., S.S., Design: K.S., S.S., Data Collection or Processing: S.S.,
Analysis or Interpretation: K.S., S.S., M.A., Literature Search: S.S., M.A., Writing: S.S., M.A.

Conflict of Interest: No conflict of interest was declared by the authors.

Financial Disclosure: The authors declared that this study received no financial support.

\section{References}

1. Kasper D, Fauci A, Hauser S, Longo D, Jameson J, Loscalzo J. Coagulation Disorders, Harrison's principles of internal medicine, 19th ed. New York, McGraw Hill Education, 2015.

2. Sanders B), Shapiro AD, Hock RA, Weddell JA, Belcher CE. Management of the medically compromised patient. McDonald and Avery Dentistry for the Child and Adolescen. $9^{\text {th }}$ ed. St. Louis, Mo, Elsevier Inc, 2011. p. 408-509.

3. Eshghi P, Mahdavi-Mazdeh M, Karimi M, Aghighi M. Haemophilia in the developing countries: The Iranian experience. Arch Med Sci 2010;6:83-9.

4. Makarem M, Talebi M, Mazhari F, Kiyanosh N. Evaluation of Oral and Dental Health Status of Khorasan Province Hemophilic Patients in 2004. J Mashhad Dent School 2008;32:161-8.

5. Fonseca MA. Oral and dental care of local and systemic diseases. Pediatric Dentistry-Infancy through Adolescence. $4^{\text {th }}$ ed. St. Louis, Mo. Elsevier Saunders, 2005. p. 74-6.

6. Kliegman RM, Behrman RE, Jenson HB, Stanton BM. Nelson textbook of pediatrics. 20 th $\mathrm{ed}$. Philadelphia, Pennsylvania, Elsevier Inc, 2016. p. 2384-9.

7. Alpkiliç Baskirt E, Ak G, Zulfikar B. Oral and general healthrelated quality of life among young patients with haemophilia. Haemophilia 2009;15:193-8.

8. Newman MG, Takei H, Klokkevold PR, Carranza FA. Carranza's Clinical Periodontology: Expert Consult. $12^{\text {th }}$ ed. St. Louis, MO. Elsevier Inc, 2014.

9. Salem K, Eshghi P. Dental health and oral health-related quality of life in children with congenital bleeding disorders. Haemophilia 2013;19:65-70.

10. Noor N, Maxood A, Mumtaz R. Dental management of haemophilic pediatric patients. Pak Oral Dent J. 2012;32.1. 